\title{
Addressing Diversity in Mindfulness Research on Health: A Narrative Review using the ADDRESSING Framework
}

\author{
Gabrielle Chin \\ Rowan University, ching4@students.rowan.edu \\ Vanessa Anyanso \\ University of Pennsy/vania, anyansov@pennmedicine.upenn.edu \\ Jeffrey Greeson \\ Rowan University, greeson@rowan.edu
}

Cooper Rowan Medical Journal: https://rdw.rowan.edu/crjcsm

Would you like to be a reviewer? Please fill in this short form to express your interest.

\section{Recommended Citation}

Chin, Gabrielle; Anyanso, Vanessa; and Greeson, Jeffrey (2019) "Addressing Diversity in Mindfulness Research on Health: A Narrative Review using the ADDRESSING Framework," Cooper Rowan Medical Journal: Vol. 1 : Iss. 1 , Article 2.

DOI: issn.2578-3343_vol1iss1.2

Available at: https://rdw.rowan.edu/crjcsm/vol1/iss1/2

\section{(c) (i)}

This work is licensed under a Creative Commons Attribution 4.0 License.

This Reviews is brought to you for free and open access by the Rowan University Journals at Rowan Digital Works. It has been accepted for inclusion in Cooper Rowan Medical Journal by an authorized editor of Rowan Digital Works. For more information, please contact brush@rowan.edu. 


\section{Addressing Diversity in Mindfulness Research on Health: A Narrative Review using the ADDRESSING Framework}

\section{Cover Page Footnote}

Competing Interests The authors declare no competing financial interests. Acknowledgments The authors thank Georita M. Frierson, Ph.D., for inspiring us to use the ADDRESSING framework as a foundation for this research. Preparation of this article was supported by the National Heart, Lung, and Blood Institute [grant numbers R01HL119977-05 and R01HL119977-05S1], the National Center for Complementary and Integrative Health [grant number U01AT009974-01], and by a fellowship from the Institute for Integrative Health (TIIH). No sponsor was involved in conceptualizing, writing, or publishing this paper. The contents of this work are solely the responsibility of the authors, and do not necessarily represent the views of the National Institutes of Health $(\mathrm{NIH})$ or $\mathrm{TIIH}$. 


\section{Addressing Diversity in Mindfulness Research on Health: A Narrative Review using the ADDRESSING Framework}

\section{Gabrielle Chin $^{1}$, Vanessa Anyanso² \& Jeffrey Greeson ${ }^{1}$}

${ }^{1}$ College of Science and Mathematics, Department of Psychology, Rowan University, Glassboro, New Jersey

${ }^{2}$ University of Pennsylvania, Philadelphia, Pennsylvannia

E-mails: ching4@students.rowan.edu (Gabrielle Chin), anyansov@pennmedicine.upenn.edu (Vanessa Anyanso), greeson@rowan.edu (Jeffrey Greeson)

\section{INTRODUCTION}

Over the past 5 years, the number of Americans practicing meditation has more than tripled, rising from $4 \%$ of adults in 2010 to $14 \%$ in $2017 .^{1}$ This rise is likely related to the increasing focus on preventive and integrative approaches to healthcare in the United States, such as meditation, whih is often used to reduce stress, anxiety, depression, and pain in conjunction with improving health and well-being. ${ }^{2}$ While many different meditative practices exist, mindfulness meditation emphasizes nonjudgmental awareness of the present moment. Although substantial research supports mindfulness-related improvements in patient-reported mental and physical health, ${ }^{3}$ the replication crisis in social science and medicine, alongside numerous methodological concerns about extant mindfulness studies, ${ }^{4}$ invites questions regarding the generalizability of research on the reported health-promoting effects of mindfulness meditation and mindfulness as an innate, dispositional quality (trait mindfulness). Moreover, as much of mindfulness research over-samples middle-to-upper class, Caucasian, women, ${ }^{5}$ the extent to which results generalize to a broader, more diverse population is unclear. One possible reason for this overrepresentation could be that this population has the time and/or finances to participate in mindfulness-based interventions (MBIs) from which researchers draw samples.

In 2001, Dr. Pamela Hays published Addressing Cultural Complexities in Practice, ${ }^{6}$ introducing the ADDRESSING framework as a guide to help clinicians better identify and understand the relevant cultural identities of their clients. According to Dr. Hays, the facets of identity include: Age, Developmental and 
acquired Disabilities, Religion, Ethnicity, Socioeconomic status, Sexual orientation, Indigenous heritage, National origin, and Gender. This framework allows room for intersectionality between identity facets and does not inherently exclude non-minority individuals. As such, the ADDRESSING framework, with its attention to multiple aspects of identity, provides an effective structure for organizing research published on different populations and identifying 1) which populations are represented and underrepresented in various categories and 2) what is known about underrepresented groups in research. The main purpose of this review, therefore, was to use the ADDRESSING framework to highlight mindfulness research conducted on historically underrepresented groups as both a method to summarize what has been done and to point out gaps for future research.

\section{METHODS}

We searched MEDLINE, PsychINFO, and Web of Science through September 2018 using the following combination of keywords: (diverse OR age OR disability OR race OR ethnicity OR Black OR White OR Latino OR Hispanic OR Asian OR gender OR transgender OR sexual orientation OR lesbian OR gay OR LGBT or LGB OR homosexual OR bisexual OR asexual OR socio-economic status OR SES OR low-income OR nationality OR indigenous OR religion OR spirituality) AND (Mindfulness OR Mindfulness-based OR MBI OR MBSR). Our search was limited to English language articles. This search string resulted in 13,626 initial hits. If the paper was a review, meta-analysis, or letter to the editor or if title or abstract did not indicate that the population sampled was historically underrepresented it was not downloaded for further review. This resulted in 1,478 articles screened for inclusion.

As the focus of this review was synthesizing recent research investigating the connection between mindfulness and mental and physical health, studies that primarily focused on brain imaging, cognitive processes, sexual response, dyadic relationships, or mechanistic questions were excluded. In addition, intervention studies were excluded that were not primarily mindfulness-based (e.g. yoga, mantra meditation). We considered including internet- and app-based mindfulness interventions. However, as mobile mindfulness interventions are not as well established in the literature, and a recent review ${ }^{7}$ of 16 web-based mindfulness intervention studies found that 14 had a majority of white, middle aged female participants, while the remaining two did not report racial demographics, we chose to exclude studies on web- and app-based interventions.

Finally, book chapters, master' s theses, dissertations, study protocols, qualitative studies, or scale validation studies were also excluded. After exclusions, 201 articles remained, which were then sorted into one of the 10 ADDRESSING categories. The articles were read in full and, to stay within journal guidelines of 50 articles, up to 5 articles from each section were chosen within each category, based on 
impact factor, methodological rigor, and cultural competency and sensitivity. Ultimately, the selection process produced the 44 articles that were included for this narrative review. See Figure 1 for a flowchart detailing this process.

Figure 1.

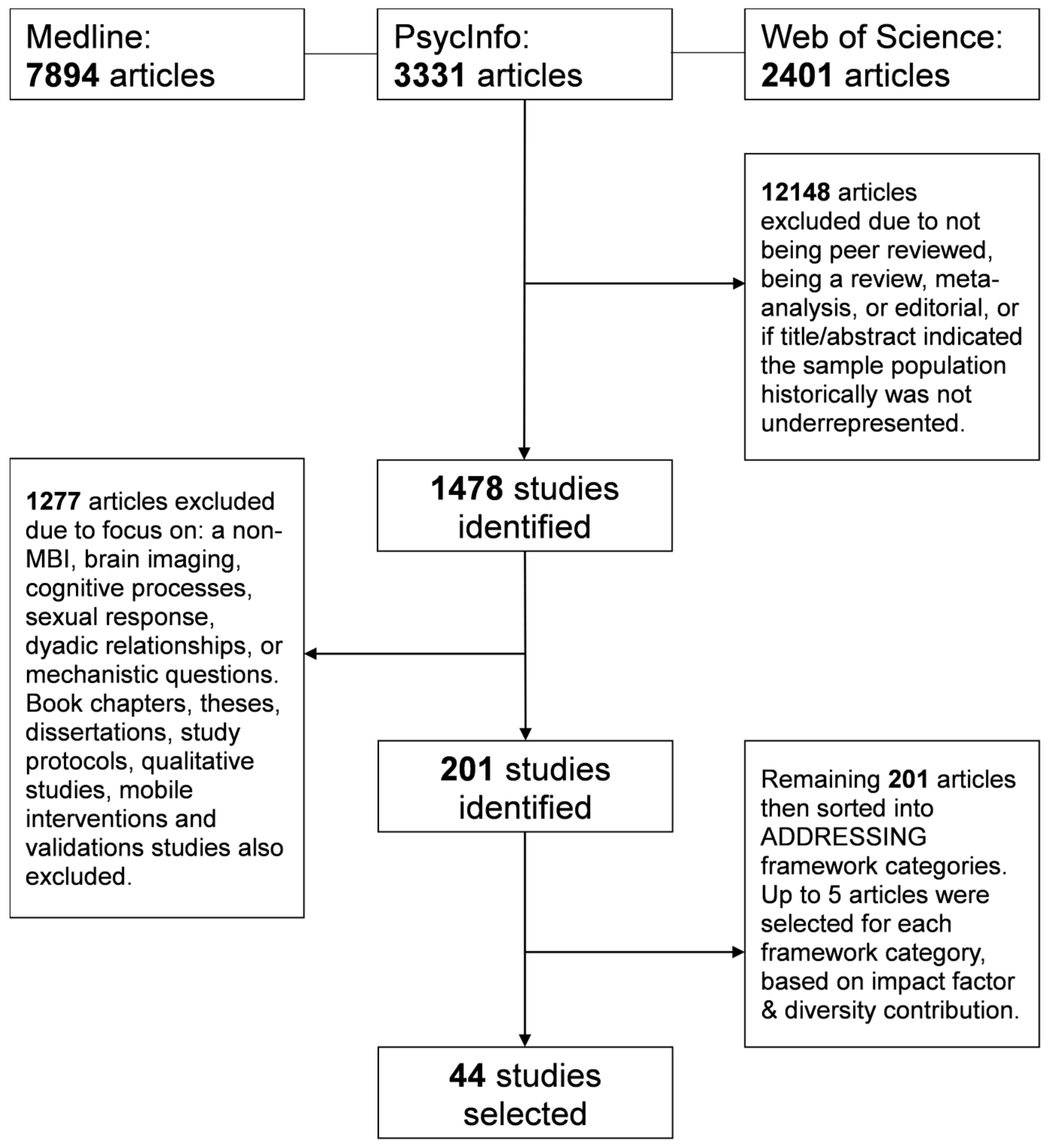

Figure 1 Article Selection Process 


\section{RESULTS}

\section{Age}

Although the bulk of mindfulness research is conducted on middle-aged adults, investigations on the impact of MBIs and trait mindfulness on health and well-being in young and older-age participants are promising. A randomized controlled trial (RCT) of stressed urban elementary and middle-school students ( $n=300, \bar{x}$ age $=12$ years) found that a 12-week Mindfulness-Based Stress Reduction (MBSR) program modified to fit into a class period and emphasize group discussion on the applications of mindfulness techniques, was acceptable and feasible. Furthermore, MBSR training in that urban youth sample significantly improved subjective measures of somatization, self-hostility, depression, rumination, negative affect, and posttraumatic stress disorder symptom severity and coping, even when compared against a health-education active control group. ${ }^{8}$ Another RCT found that kindergarteners $(n=127)$ who completed a school-based MBI showed significantly improved self-regulation skills and outsider ratings of social skills, alongside lower maladaptive impulsive, inattentive, and hyperactive behaviors, compared with participants randomized to an active control condition. Furthermore, children with baseline deficits in self-regulatory and social skills displayed greater improvement than children at average or higher-functioning levels of these domains. ${ }^{9}$ However, effectiveness of MBIs in improving facets of well-being in younger participants, particularly adolescents ( $\mathrm{n}=132 ; \overline{\mathrm{x}}$ age $=13$ years), is inconsistent, with some studies finding no effects. ${ }^{10}$ These mixed findings in children and adolescents emphasize the ongoing need to identify, replicate, and disseminate MBI formats adapted to optimally engage and benefit this population.

In older-age participants experiencing high levels of anxiety-related distress and subjective cognitive dysfunction ( $\mathrm{n}=34)$, both a traditional, 8-week MBSR training and an extended 12-week MBSR training improved subjective worry and trait mindfulness as well as objective measures of memory, with large effect sizes. However, no differences in outcomes occurred between the traditional 8-week and the extended 12-week MBSR programs, suggesting that more training is not necessarily better in older adults. ${ }^{11}$ Among a community-dwelling sample of older ( $n=117, \bar{x}$ age=68 years) and younger adults $(n=123, \bar{x}$ age=29 years), older adults demonstrated significantly greater subjective positive affect and trait mindfulness than younger participants, with trait mindfulness mediating the relationship between age and positive affect. ${ }^{12}$ Furthermore, in middle-aged and older-age adults $(n=423)$, trait mindfulness appears to buffer both adverse effects of ruminative coping with aging experiences, measured via subjective awareness of age-related gains and losses, and depression symptoms. ${ }^{13}$ In sum, studies exploring MBIs in older-age adults present generally positive well-being outcomes, and may be especially beneficial in reducing 
symptoms of depression and anxiety broadly, alongside improving anxiety and distress specifically related to the aging experience, perhaps due to the higher baseline trait mindfulness exhibited by older adults.

\section{Disability (Developmental)}

Despite initial uncertainty regarding the feasibility and effectiveness of MBIs for people with disabilities that begin early in the developmental process, early pilot and case study research tentatively suggests that tailored MBIs may improve emotion regulation, problem behaviors, and quality of life (QoL) for people with various developmental disabilities. ${ }^{14}$ For example, a recent pilot study of elementary school students with severe learning disabilities $(n=14)$ suggests a modified, 8-week Mindfulness-Based Cognitive Therapy (MBCT) training significantly improved disability-related symptoms and behaviors, including depression, anxiety, aggression and inattentiveness, with a large effect size. ${ }^{15}$ Similar results have been shown in adolescents with learning disabilities $(n=34)$, alongside improved social skills and academic success. ${ }^{16}$ In high-functioning adults with an autism spectrum disorder $(n=42)$, a 9-week MBI modified to include greater homework planning time to account for decreased executive functioning significantly improved subjective measures of affect, depression, anxiety and rumination, at medium to large effect size, versus a waitlist control group. ${ }^{17}$ Although these initial results are promising, replicability and generalizability are unclear, warranting further study. In addition, most studies of MBIs in people with developmental disabilities utilize a high-functioning sample with mild to moderate impairment. It is unknown if these beneficial outcomes would occur in people with lower levels of functioning. Still, these recent studies illustrate that MBIs reduce symptoms often co-morbid with developmental disabilities, such as depression and anxiety, and likely aid in reducing problem behaviors related to developmental disability.

\section{Disability (Acquired)}

More rigorous research exists testing the efficacy of MBIs in improving health and well-being in people with acquired disabilities, or disabilities developed later in life often resulting from accidents or illness. For example, in people on disability due to painful diabetic peripheral neuropathy ( $n=66)$, MBSR training following pain pharmacotherapy optimization decreased self-reported pain symptom severity, pain catastrophizing, and depression, alongside improved health-related QoL. ${ }^{18}$ In people with a traumatic brain injury (TBI; n=76) a 10-week RCT found that Mindfulness-Based Supportive Therapy decreased depressive symptoms compared with the waitlist control group. Furthermore, this decrease was maintained at 3-month follow-up. ${ }^{19}$ An RCT of Mindfulness-Based Alzheimer' s Stimulation (MBAS) compared to 2 active control groups, progressive muscle relaxation and cognitive stimulation therapy, significantly improved cognitive functioning in older adults $(n=120)$. Furthermore participants in the mindfulness group did not experience deterioration of cognitive abilities during the 2-year trial whereas the two active comparison groups did. ${ }^{20}$ An RCT of participants with Multiple Sclerosis experiencing low to moderately 
severe levels of disability $(\mathrm{n}=150)$ found that an 8-week MBSR training improved health-related QoL and well-being, alongside subjective measures of depression, fatigue and anxiety versus treatment as usual, at medium to large effect size. ${ }^{21}$ In smaller sample sizes, these findings have been replicated in multiple countries and expanded to include participants with peripheral neuropathy. ${ }^{22}$ Taken together, MBIs appear to improve well-being in people with acquired disabilities, perhaps more effectively than standard care interventions, and may slow deterioration of cognitive function associated with acquired disabilities.

\section{Religion}

Although our current conception of mindfulness originates from Eastern religious traditions, in the West mindfulness practice and research is primarily secular. ${ }^{23}$ Still, there is value in exploring the relationship between mindfulness, religiosity, and spirituality, given the connections of each to physical and mental health. One MBSR outcome study found significant increases in spirituality and mindfulness, and that increases in both were associated with better self-reported physical and mental health. ${ }^{24}$ Results of another MBSR outcome study found that depressive symptoms decreased similarly in both religious and nonreligious participants ${ }^{25}$ and that increased spirituality post-MBSR was directly related to increased mindfulness which, in turn, was associated with improved mental health QoL. ${ }^{26}$ Another intervention study compared a secular mindfulness training $(n=49)$ with a modified Christian MBI $(n=42)$ in college students, and found that while both groups reported decreases in depressive, anxiety, and stress scores, those in the modified Christian group reported greater reductions of distress and higher treatment adherence rates. ${ }^{27}$ Finally, a study of African-American college students $(n=184)$ found an association between religiosity and mindfulness, with those who reported being religious having higher mindfulness scores than nonreligious students. ${ }^{28}$ While increasing spirituality often is not the intent of mindfulness training in most Western research studies, it can still be a pertinent outcome for the whole-person and, for some religious individuals, grounding mindfulness in their religion can increase the benefits of mindfulness training. However, it is important to note that there is still a lack of diversity within research exploring mindfulness and religion, as samples are predominantly white and Christian.

\section{Ethnicity}

Although mindfulness practices theoretically can benefit anyone, most mindfulness research tends to involve white participants. Recently, there are a growing number of studies examining the effects of trait mindfulness and MBIs in people of color. One such study found that depressed, African-American, low-income women $(n=31)$ reported significantly decreased depressive and stress symptoms and increased self-acceptance after participating in an adapted 8-week MBSR course, with shorter sessions and content modified to be culturally relevant. ${ }^{29}$ Another study found that in a small $(n=14)$ group of male 
African-American veterans with chronic kidney disease, participants randomized to mindfulness meditation displayed significantly lower systolic blood pressure, diastolic blood pressure, mean arterial pressure, and heart rate than those in a standard blood pressure education control group. ${ }^{30}$ In a college sample ( $n=118)$ of a majority Hispanic $(n=58)$ and Black $(n=25)$ students, the observing facet of the Five-Facet Mindfulness Questionnaire was inversely related $(B=-.75, p=.03)$ to the severity of suicidal ideation; however, the four other facets of mindfulness (Describing, Non-judging, Non-reactivity, Acting with Awareness) were not related. ${ }^{31}$ A different survey of 116 Asian-American college students found that higher mindfulness scores on the Mindful Attention Awareness Scale were associated with lower scores on somatic, depressive, and anxiety scales, consistent with findings observed in primarily white student samples. ${ }^{32}$ Another study explored the relation between perceived discrimination and depressive symptoms in a diverse community sample ( $n=97$; Hispanic=37, Black=44, Asian=11), finding that high trait mindfulness served as a buffer for discrimination-related distress. ${ }^{33}$ Overall, mindfulness research involving non-white participants is becoming increasingly common and this trend should continue to ensure that established and future mindfulness interventions benefit the historically underserved. As the last study ${ }^{33}$ highlights, including research focusing on non-white participants creates room for interesting analyses, such as exploring the relationship between mindfulness, minority-status discrimination, and mental health outcomes, in addition to exploring the effect of mindfulness on traditional health outcomes, such as blood pressure or stress levels.

\section{Sexual Orientation}

Sexual orientation refers to who an individual is attracted to, irrespective of what gender they identify as. There are only a few studies which focus on the effects of mindfulness on health in sexual minority individuals. In a large RCT on predominantly gay or bisexual men $(n=177)$ living with HIV, there was no significant difference between MBSR and a disease self-management skills group on nearly all outcome measures, including psychological distress and immune markers of disease severity or progression. ${ }^{34} \mathrm{~A}$ longitudinal study focusing on gay men found that 3 types of social support (appraisal, belonging, and tangible) predicted lower psychological distress 1-year later for individuals reporting low trait mindfulness, suggesting an interaction between these constructs. ${ }^{35}$ In young women reporting same-sex attraction $(n=175)$, acceptance as measured by the Philadelphia Mindfulness Scale mediated the relationship between minority stress and depressive symptoms. ${ }^{36}$ A survey of 236 sexual minority Latinx (a gender-inclusive form of Latino/a) youth enrolled in school found that mindfulness was negatively associated with depressive symptoms, positively associated with self-esteem, and interacted with sexual orientation-based victimization to predict depressive symptoms and self-esteem. That is, associations between sexual orientation-based coping and depressive symptoms and self-esteem were only significant 
for those with low levels of mindfulness. ${ }^{37}$ Another study exploring the relationship between mindful attention, self-esteem, and psychological QoL in lesbian, gay, bisexual, and transgender participants $(n=177)$ found that mindful attention and self-esteem moderated the relationship between perceived stress and psychological QoL. Specifically, although mindful acceptance buffered the relationship between stress and psychological QoL at low-to-moderate levels of stress, it did not have an impact at higher levels of stress. ${ }^{38}$ While limited, research involving sexual minority participants tentatively suggests that mindfulness interacts with other constructs, such as self-esteem or stress, to have a positive effect of psychological well-being.

Overall there needs to be more research focusing on LGBTQIA+ individuals. Our literature search found only 6 studies that fell into the Sexuality category, compared to 20 for Ethnicity, and 70 for Age. With a single exception ${ }^{37}$, these studies mainly included white participants, although people of color make up nearly $40 \%$ of the LGBTQIA+ population. ${ }^{39}$ Thus, future research exploring the effects of mindfulness in sexual minorities needs to account for diversity within this population, through including more non-white sexual minorities and those within the spectrum that do not identify as gay or lesbian, such as asexual or bisexual individuals. We found no articles focused specifically on these populations.

\section{Socioeconomic Status}

One major barrier to mindfulness training is the high cost - both monetary and time - that can prevent lower-income individuals from attending classes and/or participating in research that requires a substantial time commitment, relative to modest compensation. Several recent studies have explored MBIs in economically disadvantaged individuals. An 8-week mindfulness, self-compassion, and yoga training decreased depression and anxiety alongside increased self-compassion and perceived wellness in non-white, low-income female participants $(n=20) .{ }^{40}$ In a low-income Belgian sample $(n=42)$ an 8 -week MBI significantly reduced symptoms of stress, anxiety, depression, cognitive reactivity, and overgeneralization along with improved mindfulness. ${ }^{41}$ Another intervention study provided MBSR training to a low income, inner-city population, giving participants $(n=68)$ the choice to take the course in English or Spanish. Researchers found that both groups reported similar improvements in 5 of the 8 measures of health-related QoL measured by the SF-36 (i.e., general health, vitality, social functioning, role limitations due to physical health, and role limitations due to emotional health). Of note, while improvements were similar between groups, the Spanish speaking group reported significantly poorer health pre- and post-intervention, suggesting that populations facing health disparities might especially benefit from mindfulness training. ${ }^{42}$ Questions of feasibility are also important in adapting MBIs to economically disadvantaged populations. In the study just described, only $66 \%$ of participants who started 
actually completed the MBSR course. A different study assessed the feasibility and acceptability of Mindfulness-Based Relapse Prevention (MBRP) for culturally diverse (Hispanic=144, Black=110), low-income women $(n=316)$. While MBRP significantly decreased stress and drug and alcohol severity, only one-third (36\%) of participants attended more than half the sessions, and 44\% did not attend any sessions at all. ${ }^{43}$

In summary, research about MBIs in low-income individuals is promising, yet questions regarding the feasibility of standard MBIs in this population need to be explored further, because effectiveness cannot be ascertained if barriers (e.g., substantial time commitment, lack of childcare, etc.) prevent many low-income individuals from participating in MBIs.

\section{Indigenous Heritage}

Few studies have examined mindfulness, or the effects of MBIs, in individuals from indigenous backgrounds. However, some recent studies evaluated the acceptability of MBIs in Native Americans, particularly in addressing high suicide rates within some tribes. An early-stage feasibility study found that mindfulness as a concept was considered generally acceptable, as mindful qualities, such nonjudgment and openness, resonated with core components of Native American spirituality common in most tribes. ${ }^{44}$ Following this finding, Le and Golbert piloted a culturally appropriate, 10-module mind-body awareness program in a Native American secondary school with students ( $n=8, \bar{x}$ age $=17$ years) from the Bitterroot Salish (Squelix' u), thePend d' Oreille (Qlispe' ), and the Kootenai (Ktunaxa) tribes. ${ }^{44}$ Idiographic analyses revealed significantly decreased reported suicidality, mind wandering, and improvement in subjective present-moment engagement, but no change on ability to focus on a task. Through qualitative components, participants reported increased ability to focus attention and improved awareness of thoughts and emotions post-intervention. The current review found a single ongoing study utilizing input from Native American tribal members to develop an acceptable and feasible MBI for improving diabetes-related health behaviors in Native American people, which could be modified to be tribe-specific. ${ }^{45}$

While initial acceptance and feasibility studies of mindfulness for Native American people exist, ethically-minded development of culturally-sensitive MBIs should occur with the inclusion of Native American people in a collaborative intervention development process. Furthermore, although the current review found only studies involving Native American participants, mindfulness researchers would likely benefit from increased inclusion of representatives from indigenous cultures around the world.

\section{National Origin}

An overwhelming amount of mindfulness research occurs within the United States. Still, research conducted outside of the United States shows that some, but not all, effects of mindfulness tested in 
American samples may generalize to other countries. The first study of MBSR efficacy completed in South Africa $(n=276$ ) revealed that MBSR training improved trait mindfulness, positive mood, and subjective medical and psychological symptoms, and decreased negative mood and stress. ${ }^{46}$ Another study examined differences in how trait mindfulness moderated the relationship between distress disclosure, life satisfaction, and depression symptoms. Specifically, for Taiwanese ( $n=256)$ but not European American $(n=209)$ participants, trait mindfulness moderated the relationship between distress disclosure, life satisfaction and depression symptoms, indicating mindfulness may aid stress regulation in Taiwanese people, specifically. ${ }^{47}$ Furthermore, utilization of MBIs varies nationally. For example, in the treatment of depression, the United Kingdom typically recommends MBCT as a freestanding intervention, while other countries, like Sweden ${ }^{48}$ and the United States ${ }^{49}$, integrate elements of mindfulness in primary care for depression. In summary, some but not all mindfulness effects and properties are generalizable across certain nationalities. Still, MBIs are increasingly utilized across the globe, and mindfulness research is geographically expanding, highlighting the need to understand acceptability and feasibility of mindfulness concepts and interventions across countries and cultures, to guide the translation and adaptation of existing MBIs.

\section{Gender}

Whereas sexual orientation refers to sexual attraction, gender refers to an individual' s self-ascribed identity. Men are underrepresented in mindfulness research, making up less than 29\% of mindfulness study participants; furthermore, very few studies have examined possible gender differences in the effects of mindfulness training. ${ }^{50}$ In one study of university students ( $\mathrm{n}=77 ; 41$ male) who participated in a 12-week MBI, female participants reported greater improvement in negative affect, which was mediated via increased mindfulness (specifically, the non-reactivity, non-judgment, and observing facets) and self-compassion. In contrast, male participants did not demonstrate improved negative affect, but did show growth in mindfulness and self-compassion measures.$^{51}$ Conversely, an earlier study that recruited individuals personally seeking to complete MBSR training ( $n=83 ; 58$ female) found substantial between-subjects differences in the relationship between subjective measures of mindfulness and negative affect, which was not explained by gender or other demographic characteristics. ${ }^{52}$ It is unclear if effects of MBIs are equal across males and females, and relatively few mindfulness studies have adequate male participant representation. Furthermore, like the scarcity of mindfulness research focused on or including trans* participants, no studies examining mindfulness in non-binary and agender people were found in the current review. Trans*, non-binary, and agender people face unique stressors, and it is unclear how people experiencing these types of stress might be affected by MBIs. 


\section{DISCUSSION}

Overall, mindfulness can reduce stress and improve mental health in diverse populations. Given the unique stressors and mental health disparities individuals in diverse groups experience, mindfulness-related changes in mental health likely support improvements in health-related behavior, QoL and well-being. Indeed, improvements in QoL and well-being are evidenced in multiple diverse populations. ${ }^{21,26,38,42}$ Although promising, this does not mean current findings are universal, given both substantial knowledge gaps within the facets of identity specified in the ADDRESSING framework, and the multifaceted, intersectional formulation of any individual identity. While the ADDRESSING framework is helpful in identifying these initial gaps in individual facets, it is possible for an individual to be represented in one category (e.g., being a woman or middle-aged) and underrepresented in another (e.g., being a black woman or being a low-income middle-aged individual). Going forward, researchers should aim to fill the discussed gaps in research, and pay increasing attention to intersections of identities

The studies reviewed suggest that small adaptations, such as infusion of religious elements into an intervention, or adjusting the length of time of a meeting to fit with the target population' s schedule, may increase acceptability and feasibility of existing MBIs in diverse populations. Modifications of existing MBIs, when guided by members of the community the researcher is seeking to study, can enhance acceptability of mindfulness practices and interventions. In addition to modifying MBIs, future research could examine the interaction of trait mindfulness with aspects of identity and reactions to identity-based discrimination. ${ }^{33,37}$ Although mindfulness practice and research is primarily secular in the West, there is still room for research about the degree to which mindfulness is utilized as a secular versus spiritual practice and whether that results in differential health outcomes. Furthermore, web- and app-based mindfulness interventions are increasingly prevalent and utilized, though only initial rigorous testing of their effects has occurred. Future development, testing, and implementation of these feasible, accessible, culturally-sensitive MBIs, including those adapted for mobile delivery, will be an essential next step to further understand the potential health benefits of mindfulness across diverse, underrepresented, and medically underserved communities.

\section{COMPETING INTERESTS}

The authors declare no competing financial interests. 


\section{REFERENCES}

1. Clarke TC, Barnes PM, Black LI, Stussman BJ, Nahin RL. Use of yoga, meditation, and chiropractors among U.S. adults aged 18 and over. NCHS Data Brief. 2018. 325.

2. Goyal M, Singh S, Sibinga EMS, et al. Meditation Programs for Psychological Stress and Well-being: A Systematic Review and Meta-analysis. JAMA Intern Med. 2014;174(3):357-368. doi:10.1001/jamainternmed.2013.13018

3. De Vibe MF, Bjørndal A, Fattah S, Dyrdal GM, Halland E, Tanner-Smith EE. Mindfulness-based stress reduction (MBSR) for improving health, quality of life and social functioning in adults: a systematic review and meta-analysis. 264.

4. Van Dam NT, van Vugt MK, Vago DR, et al. Mind the hype: A critical evaluation and prescriptive agenda for research on mindfulness and meditation. Perspect Psychol Sci. 2018;13(1):36-61. doi:10.1177/1745691617709589

5. Waldron EM, Hong S, Moskowitz JT, Burnett-Zeigler I. A Systematic Review of the Demographic Characteristics of Participants in US-Based Randomized Controlled Trials of Mindfulness-Based Interventions. Mindfulness. 2018;9(6):1671-1692. doi:10.1007/s12671-018-0920-5.

6. Hays PA. Addressing Cultural Complexities in Practice: A Framework for Clinicians and Counselors. Washington, DC, US: American Psychological Association; 2001.

7. Toivonen KI, Zernicke K, Carlson LE. Web-Based Mindfulness Interventions for People With Physical Health Conditions: Systematic Review. J Med Internet Res. 2017;19(8):e303. doi:10.2196/jmir.7487

8. Sibinga EMS, Webb L, Ghazarian SR, Ellen JM. School-based mindfulness instruction: an RCT. Pediatrics. 2016;137(1):e20152532. doi:10.1542/peds.2015-2532

9. Viglas M, Perlman M. Effects of a mindfulness-based program on young children' s self-regulation, prosocial behavior and hyperactivity. J Child Fam Stud. 2018;27(4):1150-1161. doi:10.1007/s10826-017-0971-6

10. Johnson C, Burke C, Brinkman S, Wade T. Effectiveness of a school-based mindfulness program for transdiagnostic prevention in young adolescents. Behav Res and Ther. 2016;81:1-11. doi:10.1016/j.brat.2016.03.002 
11. Lenze EJ, Hickman S, Hershey T, et al. Mindfulness-based stress reduction for older adults with worry symptoms and co-occurring cognitive dysfunction: MBSR in older adults. Int J of Geriatr Psychiatry. 2014;29(10):991-1000. doi:10.1002/gps.4086

12. Shook NJ, Ford C, Strough J, Delaney R, Barker D. In the moment and feeling good: Age differences in mindfulness and positive affect. Transl Issues in Psychol Sci. 2017;3(4):338-347. doi:10.1037/tps0000139

13. Dutt AJ, Wahl H-W, Rupprecht FS. Mindful vs. mind full: Processing strategies moderate the association between subjective aging experiences and depressive symptoms. Psychol Aging. 2018;33(4):630-642. doi:10.1037/pag0000245

14. 14. Hastings, RP, Manikam R. Mindfulness and acceptance in developmental disabilities: introduction to the special issue. Mindfulness. 2013;4(3):85-88 doi:http://dx.doi.org/10.1007/s1267 $1-013-0207-9$

15. Malboeuf-Hurtubise C, Lacourse E, Taylor G, Joussemet M, Ben Amor L. A mindfulness-based intervention pilot feasibility study for elementary school students with severe learning difficulties: effects on internalized and externalized symptoms From an emotional regulation perspective. J Evid Based Complementary Altern Med. 2017;22(3):473-481. doi:10.1177/2156587216683886

16. Beauchemin J, Hutchins TL, Patterson F. Mindfulness meditation may lessen anxiety, promote social skills, and improve academic performance among adolescents with learning disabilities. Complement Health Pract Rev. 2008;13(1):34-45. doi:

17. Spek AA, van Ham NC, Nyklícek I. Mindfulness-based therapy in adults with an autism spectrum disorder: A randomized controlled trial. Res Dev Disabil. 2013;34(1):246-253. doi:10.1016/j.ridd.2012.08.009

18. Nathan, H., Poulin, P., Wozny, D., Taljaard, M., Smyth, C., Gilron, I., Sorisky, A., Lochnan, H. and Shergill, Y.Randomized Trial of the Effect of Mindfulness-Based Stress Reduction on Pain-Related Disability, Pain Intensity, Health-Related Quality of Life, and A1C in Patients With Painful Diabetic Peripheral Neuropathy. Clinical Diabetes. 2017;35(5), pp.294-304.

19. Bédard M, Felteau M, Marshall S, et al. Mindfulness-based cognitive therapy reduces symptoms of depression in people with a traumatic brain injury: results from a randomized controlled trial. J Head Trauma Rehabil. 2014;29(4):E13-E22. doi:10.1097/HTR.0b013e3182a615a0 
20. Quintana-Hernández DJ, Miró-Barrachina MT, Ibáñez-Fernández IJ, et al. Mindfulness in the maintenance of cognitive capacities in Alzheimer' s Disease: A randomized clinical trial. J Alzheimers Dis. 2016;50(1):217-232. doi:

21. Grossman P, Kappos L, Gensicke H, et al. MS quality of life, depression, and fatigue improve after mindfulness training. Neurology. 2010;75(13):1141-1149.

22. Tavee J, Rensel M, Planchon SM, Butler RS, Stone L. Effects of Meditation on pain and quality of life in multiple sclerosis and peripheral neuropathy. Int J MS Care. 2011;13(4):163-168. doi:10.7224/1537-2073-13.4.163

23. Compson, J.F. Is mindfulness secular or religious, and does it matter?. In Practitioner' s Guide to Ethics and Mindfulness-Based Interventions. Cham, Switzerland: Springer International Publishing; 2017.

24. Carmody J, Reed G, Kristeller J, Merriam P. Mindfulness, spirituality, and health-related symptoms. J Psychosom Res. 2008;64(4):393-403. doi:10.1016/j.jpsychores.2007.06.015

25. Greeson JM, Smoski MJ, Suarez EC, et al. Decreased symptoms of depression after Mindfulness-Based Stress Reduction: potential moderating effects of religiosity, spirituality, trait mindfulness, sex, and age. J Altern and Complement Med. 2015;21(3):166-174. doi:10.1089/acm.2014.0285

26. Greeson JM, Webber DM, Smoski MJ, et al. Changes in spirituality partly explain health-related quality of life outcomes after mindfulness-based stress reduction. J Behav Med. 2011;34(6):508-518. doi:10.1007/s10865-011-9332-x

27. Ford K, Garzon F. Research note: A randomized investigation of evangelical Christian accommodative mindfulness. Spiritual Clin Pract. 2017;4(2):92-99. doi:10.1037/scp0000137

28. Spofford JL, Nevels RM, Gontkovsky ST, Bell TP. Meditative practices predict spirituality but mindfulness does not predict alcohol use in African-American college students. Ment Health Relig Cult. 2014;17(4):379-389. doi:10.1080/13674676.2013.810614

29. Burnett-Zeigler IE, Satyshur MD, Hong S, Yang A, T. Moskowitz J, Wisner KL. Mindfulness based stress reduction adapted for depressed disadvantaged women in an urban Federally Qualified Health Center. Complement Ther in Clin Pract. 2016;25:59-67. doi:10.1016/j.ctcp.2016.08.007 
30. Park J, Lyles RH, Bauer-Wu S. Mindfulness meditation lowers muscle sympathetic nerve activity and blood pressure in African-American males with chronic kidney disease. Am J Physiol Regul Integr Comp Physiol. 2014;307(1):R93-R101. doi:10.1152/ajpregu.00558.2013

31. Chesin MS, Jeglic EL. Factors associated with recurrent suicidal ideation among racially and ethnically diverse college students with a history of suicide attempt: the role of mindfulness. Arch Suicide Res. 2016;20(1):29-44. doi:10.1080/13811118.2015.1004488

32. Masuda A, Mandavia A, Tully EC. The role of psychological inflexibility and mindfulness in somatization, depression, and anxiety among Asian Americans in the United States. Asian Am J Psychol. 2014;5(3):230-236. doi:10.1037/a0034437

33. Shallcross AJ, Spruill TM. The protective role of mindfulness in the relationship between perceived discrimination and depression. Mindfulness. 2018;9(4):1100-1109. doi:10.1007/s12671-017-0845-4

34. Hecht FM, Moskowitz JT, Moran P, et al. A randomized, controlled trial of mindfulness-based stress reduction in HIV infection. Brain Behav Immun. 2018;73:331-339. doi:10.1016/j.bbi.2018.05.017

35. Lyons A, Alba B, Pepping CA. The impact of social support and mindfulness on the mental health of middle-aged and older gay men: A longitudinal cohort analysis. Psychol Sex Orientat Gend Divers. 2017;4(4):472-480. doi:10.1037/sgd0000247

36. Bergfeld JR, Chiu EY. Mediators in the relationship between minority stress and depression among young same-sex attracted women. Prof Psychol Res Pr. 2017;48(5):294-300. doi:10.1037/pro0000155

37. Toomey RB, Anhalt K. Mindfulness as a coping strategy for bias-based school victimization among Latina/o sexual minority youth. Psychol Sex Orientat Gend Divers. 2016;3(4):432-441. doi:10.1037/sgd0000192

38. Vosvick M, Stem W. Psychological quality of life in a lesbian, gay, bisexual, transgender sample: Correlates of stress, mindful acceptance, and self-esteem. Psychol Sex Orientat Gend Divers September 2018. doi:10.1037/sgd0000303

39. Same-sex couple and LGBT demographic data interactive. The Williams Institute, UCLA School of Law. https://williamsinstitute.law.ucla.edu/visualization/lgbt-stats/?topic=LGBT\#density. Published May 2016. Accessed November 29, 2018. 
40. Falsafi N, Leopard L. Pilot study: use of mindfulness, self-compassion, and yoga practices with low-income and/or uninsured patients with depression and/or anxiety. J Holist Nurs. 2015;33(4):289-297.

41. Van der Gucht K, Takano K, Van Broeck N, Raes F. A mindfulness-based intervention for economically disadvantaged people: effects on symptoms of stress, anxiety, and depression on cognitive reactivity and overgeneralization. Mindfulness. 2015;6(5):1042-1052. doi:10.1007/s12671-014-0353-8

42. Roth B, Robbins D. Mindfulness-based stress reduction and health-related quality of life: findings from a bilingual inner-city patient population: Psychosom Med. 2004;66(1):113-123. doi:10.1097/01.PSY.0000097337.00754.09

43. Amaro H, Spear S, Vallejo Z, Conron K, Black DS. Feasibility, acceptability, and preliminary outcomes of a mindfulness-based relapse prevention intervention for culturally-diverse, low-income women in substance use disorder treatment. Subst Use Misuse. 2014;49(5):547-559. doi:10.3109/10826084.2013.852587

44. Le TN, Gobert JM. Translating and implementing a mindfulness-based youth suicide prevention intervention in a Native American community. J Child Fam Stud. 2015;24(1):12-23. doi:10.1007/s10826-013-9809-z

45. Proulx J. Exploring the adaption of mindfulness in Native American communities to address diabetes.

46. Whitesman SL, Hoogenhout M, Kantor L, Leinberger KJ, Gevers A. Examining the impact of a mindfulness-based stress reduction intervention on the health of urban South Africans. Afr J Prim Health Care Fam Med. 2018;10(1).

47. Chien WT, Bressington D, Yip A, Karatzias T. An international multi-site, randomized controlled trial of a mindfulness-based psychoeducation group programme for people with schizophrenia. Psychol Med. 2017;47(12):2081-2096. doi:10.1017/S0033291717000526

48. Lilja JL, Zelleroth C, Axberg U, Norlander T. Mindfulness-based cognitive therapy is effective as relapse prevention for patients with recurrent depression in Scandinavian primary health care. Scand J Psychol. 2016;57(5):464-472. doi:10.1111/sjop.12302

49. Glover NG, Sylvers PD, Shearer EM, et al. The efficacy of focused acceptance and commitment therapy in VA primary care. Psychol Serv. 2016;13(2):156-161. doi:10.1037/ser0000062 
50. Bodenlos JS, Strang K, Gray-Bauer R, Faherty A, Ashdown BK. Male representation in randomized clinical trials of mindfulness-based therapies. Mindfulness. 2017;8(2):259-265.

51. Rojiani R, Santoyo JF, Rahrig H, Roth HD, Britton WB. Women benefit more than men in response to college-based meditation training. Front Psychol. 2017;8.

52. Snippe E, Nyklícek I, Schroevers MJ, Bos EH. The temporal order of change in daily mindfulness and affect during mindfulness-based stress reduction. J Couns Psychol. 2015;62(2):106-114.

\section{ACKNOWLEDGMENTS}

The authors thank Georita M. Frierson, Ph.D., for inspiring us to use the ADDRESSING framework as a foundation for this research. Preparation of this article was supported by the National Heart, Lung, and Blood Institute [grant numbers R01HL119977-05 and R01HL119977-05S1], the National Center for Complementary and Integrative Health [grant number U01AT009974-01], and by a fellowship from the Institute for Integrative Health (TIIH). No sponsor was involved in conceptualizing, writing, or publishing this paper. The contents of this work are solely the responsibility of the authors, and do not necessarily represent the views of the National Institutes of Health (NIH) or TIIH. 